\title{
Development of a Single In Vitro Dissolution Method for a Combination Trilayer Tablet Formulation of Clopidogrel and Pravastatin
}

\author{
Zongyun Huang ${ }^{1, *}$, Ruben Lozano ${ }^{1}$, Robert Francis' ${ }^{1}$, Anne-Françoise \\ Aubry ${ }^{2}$, Alyson Steckbeck ${ }^{1}$, and Denis O. Sciascia ${ }^{1}$ \\ 'Bristol-Myers Squibb, Research and Development, 1 Squibb Drive, New Brunswick, NJ 08901, USA \\ ${ }^{2}$ Bristol-Myers Squibb, Research and Development, Route 206 and Province Line Road, \\ Lawrenceville, New Jersey 08540, USA
}

e-mail:zongyun.huang@bms.com

\begin{abstract}
A fixed-combination dose, trilayer tablet formulation was developed for two drugs already marketed as individual products, Plavix in which clopidogrel, an anti-clotting agent, is the active ingredient and pravastatin, an HMG-CoA reductase inhibitor for the treatment of hypercholesterolemia. To simplify quality control testing, the preference is to use a single dissolution method for the analysis of multiple active components in a combination tablet. However, development of one dissolution method for clopidogrel and pravastatin is particularly challenging because of the divergent $\mathrm{pH}$ solubility and $\mathrm{pH}$ dependent stability of these two drugs. At low $\mathrm{pH}(<3)$, clopidogrel bisulfate is most soluble and stable, whereas pravastatin sodium rapidly degrades due to lactonization and oxidation. Conversely, at a neutral pH and higher, pravastatin sodium is most soluble and stable, but clopidogrel bisulfate undergoes hydrolysis and racemization. This article describes the development of a single dissolution method to accommodate both drugs, including selection of medium $\mathrm{pH}$ and surfactant. The method uses USP Apparatus 2 (paddles) at $75 \mathrm{rpm}$ in $1000 \mathrm{~mL}$ of citrate buffer (0.05 M, $\mathrm{pH}$ 5.5) medium containing $2 \%$ CTAB (cetyltrimethyl ammonium bromide, a cationic surfactant) at $37^{\circ} \mathrm{C}$. This dissolution methodology provides good dissolution profiles for both clopidogrel and pravastatin and is able to discriminate the changes in composition, manufacturing process, and stability for the combination tablets. To quantitate both drugs simultaneously, a rapid isocratic reversed-phase liquid chromatographic method was developed and validated.
\end{abstract}

\section{INTRODUCTION}

A fixed-dose combination tablet of clopidogrel and pravastatin was developed for the prevention of cardiovascular events in patients with heart disease or hypercholesterolemia. Although an aspirinpravastatin combination product is on the market for the same indication (1-3), clopidogrel is more effective than aspirin in reducing the combined risk of ischemic stroke, myocardial infarction, or vascular death due to the mechanistic difference. In addition, clopidogrel has little ulcerogenic effect because it does not cause gastric mucosal injury by direct exposure (4-5).

A trilayer tablet formulation was chosen for development because of the incompatibility of the two drugs. The layers containing clopidogrel and pravastatin are separated by an inert layer. To support the product development effort, a suitable dissolution methodology, preferably a single method for both actives, was essential.

Clopidogrel bisulfate (Figure $1 \mathrm{~A}$ ) is an adenosine diphosphate (ADP) receptor inhibitor that prevents platelets in the blood from sticking together and forming clots (6). A summary of the clopidogrel solubility data shown in Table 1 reveals that it is fairly soluble and stable in aqueous solution at low $\mathrm{pH}$; however, the solubility

${ }^{*}$ Corresponding author. drops steeply when the solution $\mathrm{pH}$ is above 3. Pravastatin sodium (Figure 1B) is an HMG CoA reductase inhibitor, a class of lipid-lowering compounds that reduce cholesterol biosynthesis (7). Pravastatin sodium is the most soluble and stable at neutral $\mathrm{pH}$ and higher but degrades via lactonization and oxidation at about $\mathrm{pH} 4$ and lower.

Dissolution methods for single-entity products of clopidogrel and pravastatin have been filed with various regulatory agencies. Developing a dissolution method for a combination product of these two drugs was particularly challenging because of their divergent $\mathrm{pH}$-dependent solubility and stability. The main challenge was to find a dissolution medium to accommodate the two drugs. At low pH, clopidogrel bisulfate is most soluble, but pravastatin sodium degrades rapidly. Staying within the $\mathrm{pH}$ range of 4.5-6.8 provides adequate stability of pravastatin and clopidogrel as well as good pravastatin solubility. However, clopidogrel exhibits poor dissolution in this $\mathrm{pH}$ range.

In addition, sample analysis by direct spectrophotometry was unsuitable for the combination product owing to the lack of specificity. Several high performance liquid chromatographic (HPLC) methods have been reported for the separation and quantitation of clopidogrel or pravastatin in biological fluids and drug product (8-13). However, none of these methods was used for the 
<smiles></smiles>

(A)<smiles>CCC(C)(C)C(=O)OC1CC(O)C=C2C=CC(C)C(CCC(O)CC(O)CC(=O)O[NH3+])C21</smiles>

(B)

Figure 1. The chemical structures of $(A)$ clopidogrel bisulfate and (B) pravastatin sodium.

simultaneous determination of the compounds in a combination product. A gradient HPLC procedure to determine clopidogrel, pravastatin, and their impurities and degradation products simultaneously was developed

Table 1. Solubility Data for Clopidogrel and Pravastatin

\begin{tabular}{lcc}
\hline \multicolumn{2}{l}{ Clopidogrel Solubility at $\mathbf{2 5}{ }^{\circ} \mathrm{C}$} \\
\hline Initial $\mathbf{~ H H}$ & Final $\mathbf{~ H ~}$ & $\mathbf{~ m g / m L ~ D i s s o l v e d ~}$ \\
\hline 2.6 & 2.2 & 6.80 \\
\hline 3.0 & 2.5 & 3.00 \\
\hline 4.0 & 4.1 & 0.05 \\
\hline 6.0 & 5.7 & 0.01 \\
\hline 8.0 & 7.8 & 0.01 \\
\hline
\end{tabular}

Pravastatin Solubility at $37^{\circ} \mathrm{C}$

\begin{tabular}{lc}
\hline Solvent Parameters & $\mathbf{m g} / \mathbf{m L}$ Dissolved \\
\hline Water & $>1000$ \\
\hline pH 1.2 Simulated Gastric Fluid (no enzyme) & 8 \\
\hline pH 7.5 Simulated Intestinal Fluid (no enzyme) & $>1000$ \\
\hline
\end{tabular}

internally. However, for dissolution sample analysis, an isocratic HPLC method that provides adequate specificity of the two analytes while maintaining a reasonable run time was preferred. Therefore, development of a rapid HPLC method for the simultaneous determination of clopidogrel and pravastatin in the dissolution samples was pursued.

This paper describes the development and validation of a single dissolution method and HPLC analysis for dosage forms containing a combination of clopidogrel and pravastatin.

\section{EXPERIMENTAL \\ Materials}

The combination tablets containing clopidogrel and pravastatin were provided by Bristol-Myers Squibb Biopharmaceutics R\&D (New Brunswick, NJ). The target potencies were $75 \mathrm{mg}$ for clopidogrel as free base and two strengths of 80 and $20 \mathrm{mg}$ for pravastatin as free acid. The total tablet weights were approximately $875 \mathrm{mg}$ for the 75/80-mg dose and $435 \mathrm{mg}$ for the 75/20-mg dose. The clopidogrel bisulfate and pravastatin (sodium salt) reference standards were obtained from Bristol-Myers Squibb Process R\&D.

Reagent grade chemicals were used unless otherwise indicated. Hydrochloric acid (0.1 N), glacial acetic acid, $10 \mathrm{~N}$ sodium hydroxide (J.T.Baker, Phillipsburg, NJ), potassium phosphate monobasic, sodium phosphate monobasic, citric acid, sodium citrate dihydrate, and sodium acetate (EM Science, Gibbstown, NJ) were used to prepare the dissolution media. Sodium dodecyl sulfate (SDS, EM Science), octyl phenol ethoxylate (J.T.Baker), and cetyltrimethyl ammonium bromide (CTAB, Sigma-Aldrich, St. Louis, MO) were used as surfactant additives to the medium. Barnstead purified water (Barnstead International, Dubuque, IA) was used to prepare both dissolution media and HPLC mobile phases. Trifluoroacetic acid and HPLC grade methanol and acetonitrile (J.T.Baker) were used for the preparation of the mobile phases.

\section{Reagents}

The sodium acetate buffer ( $\mathrm{pH} 4.5,0.05 \mathrm{M})$ and potassium phosphate-sodium phosphate buffer ( $\mathrm{pH} 6.8$, $0.05 \mathrm{M}$ ) were prepared according to USP (14). Sodium citrate buffers ( $\mathrm{pH} 4.5-5.5)$ were prepared by dissolving $5.22 \mathrm{~g}$ of citric acid and $7.39 \mathrm{~g}$ of anhydrous sodium citrate per liter of water. The $\mathrm{pH}$ was adjusted to the desired value with $10 \mathrm{~N}$ sodium hydroxide. The media containing surfactant were prepared by dissolving the surfactants in the buffer.

The stock standard solutions of clopidogrel and pravastatin were prepared in methanol at concentrations of approximately $1 \mathrm{mg} / \mathrm{mL}$ and $0.5 \mathrm{mg} / \mathrm{mL}$, respectively. These stock solutions were combined and diluted to prepare clopidogrel-pravastatin working standard solutions at concentrations of approximately $0.1 / 0.08$ and $0.1 / 0.02 \mathrm{mg} / \mathrm{mL}$ for the $75 / 80$ - and $75 / 20-\mathrm{mg}$ potencies, respectively. 


\section{Dissolution Test Conditions}

Dissolution testing was performed in compliance with USP (15). The final method conditions were Apparatus 2 at a paddle speed of $75 \mathrm{rpm}, 1-\mathrm{L}$ glass vessels, and a dissolution medium of 1- $\mathrm{L}$ citrate buffer $(\mathrm{pH} 5.5 ; 50 \mathrm{mM})$ with $2 \%$ CTAB $(\mathrm{w} / \mathrm{v})$ maintained at $37 \pm 0.5^{\circ} \mathrm{C}$. Tests were conducted with one of two systems: (1) Distek Premiere 5100 with Distek autosampler (Distek, North Brunswick, NJ) or (2) Zymark Multidose G3 Automation Dissolution Work Station coupled with Vankel VK 7000 dissolution bath and Caliper MultiFill sample collection module (all from Caliper Life Sciences, Hopkinton, MA). A sample volume of $1.5 \mathrm{~mL}$ was filtered and collected directly into a 2-mL HPLC vial. Two types of filter were used for sample

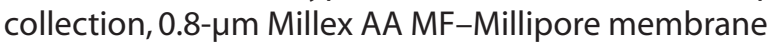

(mixed cellulose ester) filter for the Distek dissolution system and 0.45- $\mu \mathrm{m}$ Millipore PVDF filter for the Zymark Multidose automated system (both from Millipore Corp., Billerica, MA). The samples were collected at time points of $10,15,20,30,45$, and 60 min.

\section{HPLC Analysis}

For the analysis of the dissolution samples, an HPLC method with UV detection was selected because of its ability to separate clopidogrel and pravastatin from each other and from the tablet excipients and medium additives within a reasonably short time. A Waters Alliance HPLC system (Waters Corporation, Milford, MA) was used. Chromatographic data were recorded and processed using Waters Millennium software. The final

Table 2. Screening Study Results for Dissolution of Clopidogrel-Pravastatin Combination Tablets (75/80 mg) Using USP Apparatus $2(n=6)$

\begin{tabular}{|c|c|c|c|c|c|c|c|c|c|c|c|c|}
\hline & \multicolumn{6}{|c|}{$\%$ of Clopidogrel Label Dissolved } & \multicolumn{6}{|c|}{$\%$ of Pravastatin Label Dissolved } \\
\hline & \multicolumn{6}{|c|}{ Time (min) } & \multicolumn{6}{|c|}{ Time (min) } \\
\hline & 10 & 15 & 20 & 30 & 45 & 60 & 10 & 15 & 20 & 30 & 45 & 60 \\
\hline 50 rpm, $0.1 \mathrm{~N} \mathrm{HCl}(\mathrm{pH} 1.1)$ & 46 & 66 & 81 & 96 & 97 & 97 & * & * & * & * & * & * \\
\hline 50 rpm, pH 3.0 citrate buffer & 40 & 58 & 71 & 82 & 85 & 85 & 66 & 80 & 83 & 87 & 84 & 80 \\
\hline \multicolumn{13}{|l|}{50 rpm, pH 4.5 acetate buffer } \\
\hline 50 rpm, no surfactant & 26 & 36 & 41 & 43 & 43 & 43 & 77 & 90 & 95 & 98 & 98 & 100 \\
\hline 50 rpm, $1 \%$ SDS & 43 & 62 & 78 & 94 & 96 & 96 & 73 & 81 & 80 & 77 & 73 & 71 \\
\hline \multicolumn{13}{|l|}{ pH 6.8 phosphate buffer } \\
\hline 75 rpm, $1 \%$ SDS & 37 & 55 & 77 & 89 & 93 & 95 & 54 & 77 & 94 & 97 & 97 & 97 \\
\hline 75 rpm, 1\% Triton & 38 & 56 & 66 & 78 & 84 & 85 & 42 & 60 & 75 & 96 & 105 & 105 \\
\hline 75 rpm, 2\% Triton & 47 & 66 & 76 & 85 & 90 & 91 & 42 & 60 & 75 & 94 & 104 & 104 \\
\hline \multicolumn{13}{|l|}{ Acetate buffer, $2 \%$ Triton } \\
\hline 75 rpm, pH 4.5 & 42 & 59 & 74 & 87 & 92 & 93 & 53 & 73 & 86 & 100 & 100 & 101 \\
\hline 75 rpm, pH 5.0 & 52 & 71 & 81 & 88 & 93 & 94 & 47 & 64 & 78 & 97 & 101 & 102 \\
\hline 75 rpm, pH 5.5 & 35 & 50 & 65 & 80 & 84 & 86 & 52 & 72 & 88 & 102 & 103 & 102 \\
\hline \multicolumn{13}{|l|}{ Citrate buffer, $2 \%$ Triton } \\
\hline 75 rpm, pH 4.5 & 39 & 59 & 69 & 84 & 89 & 90 & 52 & 73 & 86 & 99 & 100 & 101 \\
\hline 75 rpm, pH 5.0 & 52 & 71 & 81 & 88 & 93 & 94 & 47 & 64 & 78 & 97 & 101 & 102 \\
\hline 75 rpm, pH 5.5 & 35 & 50 & 65 & 80 & 84 & 86 & 52 & 72 & 88 & 102 & 103 & 102 \\
\hline \multicolumn{13}{|l|}{ Citrate buffer, 2\%CTAB } \\
\hline 75 rpm, pH 4.5 & 32 & 47 & 63 & 88 & 96 & 98 & 41 & 57 & 73 & 95 & 98 & 98 \\
\hline 75 rpm, pH 5.0 & 29 & 44 & 57 & 83 & 96 & 99 & 42 & 59 & 72 & 92 & 98 & 97 \\
\hline $75 \mathrm{rpm}, \mathrm{pH} 5.5$ & 38 & 58 & 71 & 85 & 94 & 98 & 46 & 63 & 76 & 92 & 99 & 99 \\
\hline
\end{tabular}

* The pravastatin content could not be quantified because of the severe degradation in $0.1 \mathrm{~N} \mathrm{HCl}$. 
reversed-phase HPLC method utilized a Phenomenex Synergi Hydro-RP column $(4 \mu \mathrm{m}, 5 \mathrm{~cm} \times 4.6 \mathrm{~mm}$ i.d.; Phenomenex, Inc., Torrance, CA) maintained at $30{ }^{\circ} \mathrm{C}$ with a mobile phase of water-methanol-trifluoroacetic acid $(45: 55: 0.025 \mathrm{v} / \mathrm{v} / \mathrm{v})$, a flow rate of $1.0 \mathrm{~mL} / \mathrm{min}$, UV detection at $238 \mathrm{~nm}$, an injection volume of $10 \mu \mathrm{L}$, and a run time of $7 \mathrm{~min}$.

\section{Method Validation}

The specificity of the method was evaluated by injecting a combined solution of clopidogrel and pravastatin along with a placebo solution prepared from a blend of the tablet excipients. Linearity was established over the range of $10-200 \%$ of the nominal assay concentration for clopidogrel and $6.25-125 \%$ for pravastatin. The accuracy and precision of the method was evaluated at 80,100 , and $120 \%$ of the nominal assay concentration for clopidogrel and 75,100 , and $125 \%$ for pravastatin. Injection repeatability, effect of filtration, and solution stability were also evaluated for both clopidogrel and pravastatin in dissolution medium.

\section{RESULTS AND DISCUSSION}

\section{Development of Dissolution Methodology}

The selection of dissolution methodology was based on screening studies using USP Apparatus 2 at paddle speeds of 50 and 75 rpm; these data are listed in Table 2. The large amount of excipients in the tablets (approximate total tablet weights of 875 and $435 \mathrm{mg}$ ) resulted in coning effects, which caused high intervessel variability in the release of both drugs, particularly at a paddle speed of $50 \mathrm{rpm}$. A paddle speed of $75 \mathrm{rpm}$, which helped to minimize coning effects and high variability, was ultimately selected for the final method.

Selection of a dissolution medium to accommodate adequate solubility and stability of both clopidogrel and pravastatin was critical for this dissolution method. Several compendial dissolution media were screened; these included $0.1 \mathrm{~N}$ hydrochloric acid, sodium citrate buffer ( $\mathrm{pH}$ 3.0), and sodium acetate buffer ( $\mathrm{pH} 4.5)$. However, the results showed that pravastatin had degraded significantly before the end of the dissolution test in the media of $\mathrm{pH} 4$ or below, and clopidogrel showed poor solubility in the media above $\mathrm{pH}$ 3. Therefore, the compendial conditions were unsuitable for the combination tablets. $\mathrm{A} \mathrm{pH}$ range of 4.5-6.8 provided adequate stability of pravastatin and clopidogrel as well as good pravastatin solubility. However, since clopidogrel exhibits low solubility in this $\mathrm{pH}$ range, the addition of surfactants was subsequently investigated.

Addition of SDS in the range of $0.2-1.5 \%(\mathrm{w} / \mathrm{v})$ was investigated. Media containing SDS provided acceptable solubility for clopidogrel but appeared to contribute to more rapid degradation of pravastatin. At the end of the dissolution run, approximately $20 \%$ pravastatin degradation was observed in $\mathrm{pH} 4.5$ medium and $8 \%$ in pH 6.8 medium. Therefore, SDS is not a suitable surfactant for this application. A pH 6.8 medium containing $0.5-2 \%$
Triton X-100 (v/v) provided a slower but acceptable dissolution rate for pravastatin versus those containing SDS. However, clopidogrel solubility was still low, and high intervessel variability was observed using this medium. Sodium acetate and citrate buffers containing Triton X-100 were also investigated, but the clopidogrel dissolution rate was not improved. CTAB at $2 \%(\mathrm{w} / \mathrm{v})$ improved the dissolution rates of both drugs in the $\mathrm{pH}$ range of 4.5-5.5 with acceptable intervessel variability. A final medium $\mathrm{pH}$ of 5.5 was chosen mainly to reduce the pravastatin degradation.

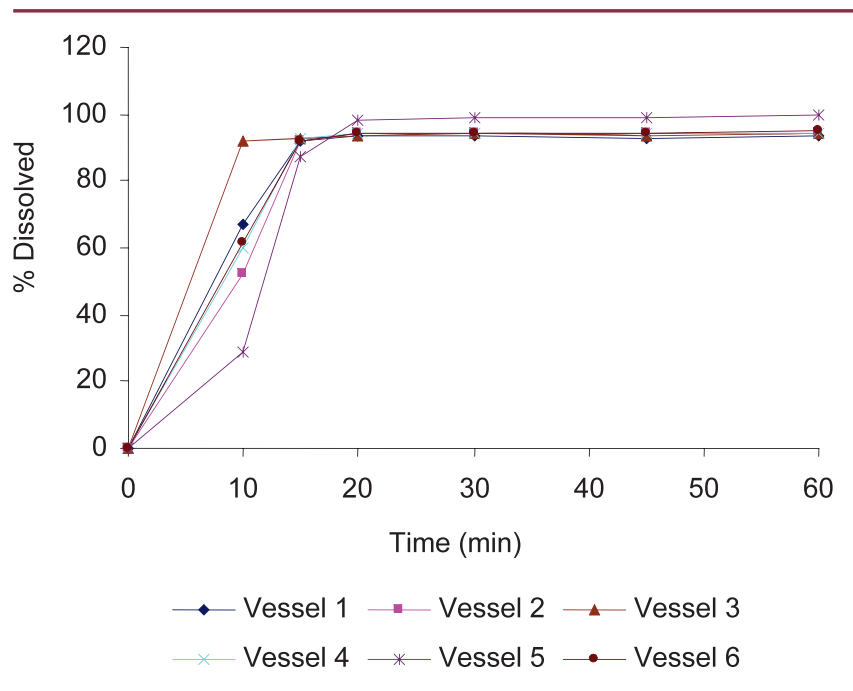

(A)

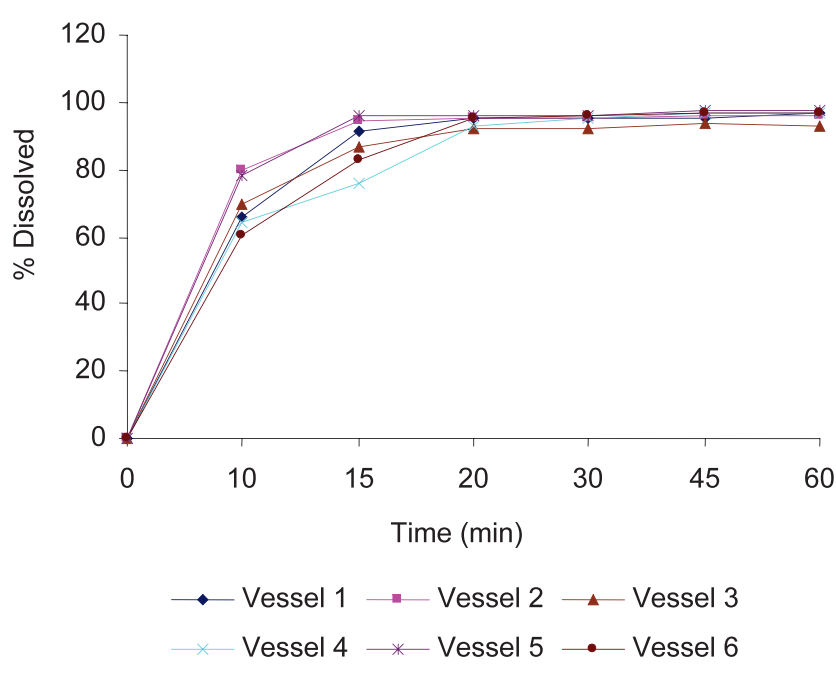

(B)

Figure 2. Effect of tablet orientation and use of sinkers on dissolution profiles of pravastatin $(20 \mathrm{mg})$ : (A) profile of tablet only; (B) profile of tablet with sinker. 
Visual observation of the tablet during dissolution suggested that the orientation of the trilayer tablets in the dissolution vessel might contribute to the intervessel variability of pravastatin dissolution, particularly for 75/20-mg tablets. The thin layer of pravastatin separated from the rest of the tablet and moved freely in the vessel over different times (from 1 to $10 \mathrm{~min}$ ) depending on tablet orientation. This variation was not significant for 75/80-mg tablets because the pravastatin layer was heavier and stayed at the bottom of the vessel after separation. A preliminary investigation was conducted using 3-prong magnetic sinkers. As shown in Figure 2,

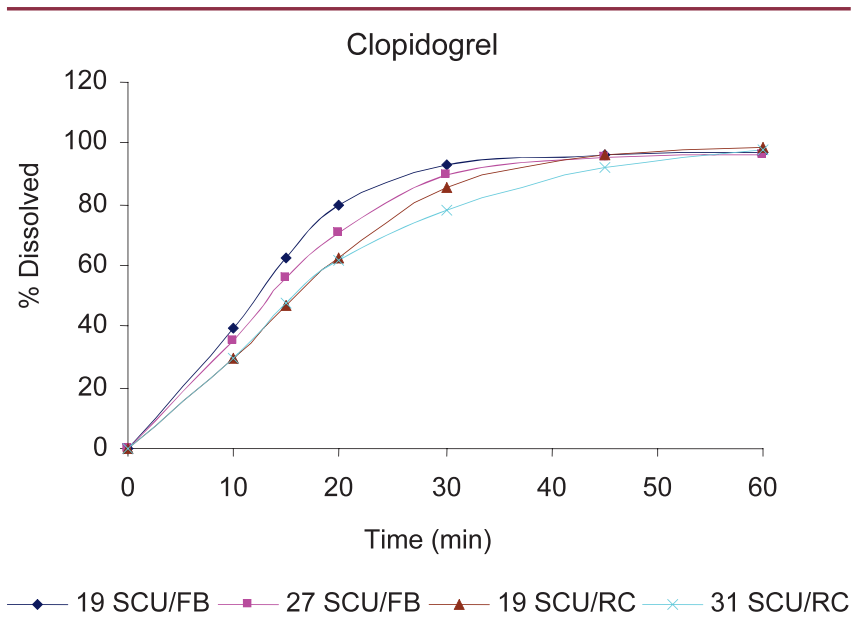

(A)

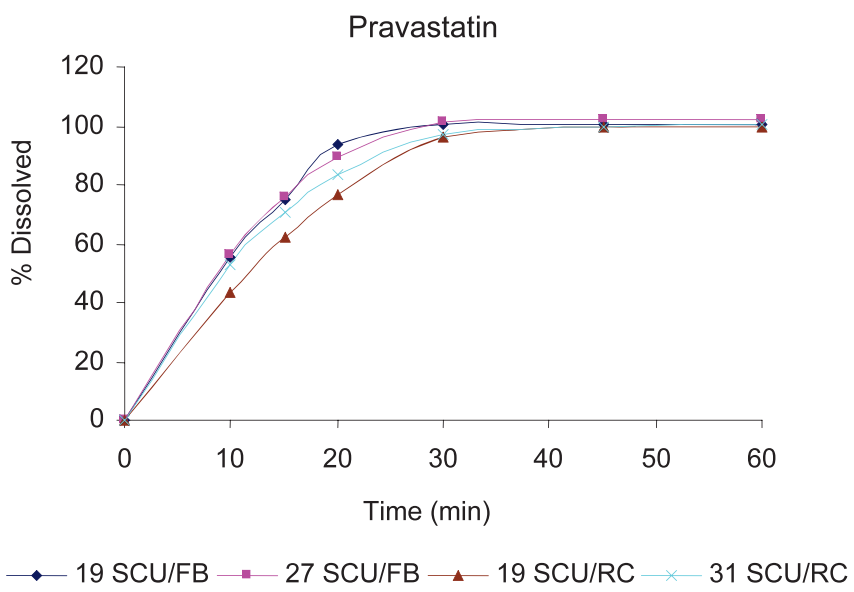

(B)

Figure 3. Comparison of dissolution profiles of clopidogrel-pravastatin combination tablets $(75 / 80 \mathrm{mg})$ manufactured with different granulation processes and compression forces (FB: fluid-bed granulation; RC: roller compactor granulation; SCU: Strong-Cobb units). (A) Profile for clopidogrel; (B) profile for pravastatin. the variation of pravastatin dissolution was reduced with use of the sinker, presumably because it eliminated direct contact between the tablets and the vessel wall, resulting in a more consistent flow of media around the tablet. Although the tablet orientation factor should be considered, it only affected the readings at the earliest time points and did not cause variability for the dissolution tests. Therefore, no sinker was used in the selected dissolution test conditions: USP Apparatus 2 (paddle) at $75 \mathrm{rpm}$ in a medium of $1000 \mathrm{~mL}$ of $0.05 \mathrm{M}$ citrate buffer, $\mathrm{pH} 5.5$, containing $2 \%$ hexadecyl trimethyl ammonium bromide (CTAB).

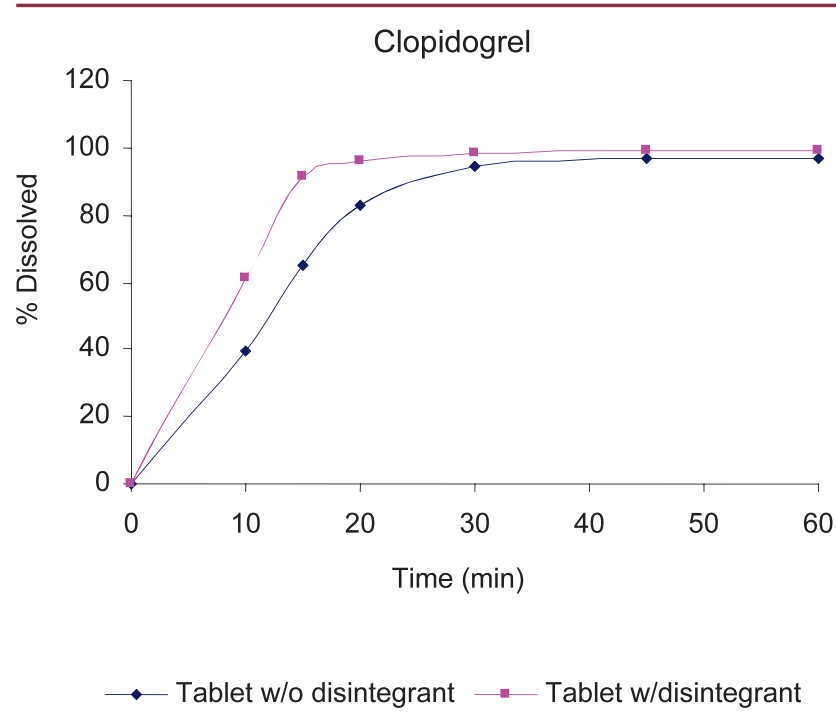

(A)

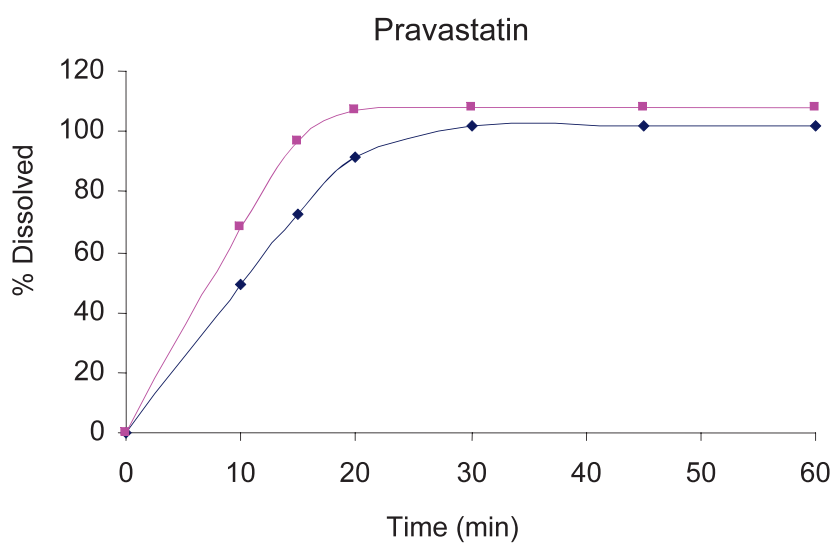

— Tablet w/o disintegrant $\_$Tablet w/disintegrant

(B)

Figure 4. Comparison of dissolution profiles of clopidogrel-pravastatin combination tablets $(75 / 80 \mathrm{mg})$ formulated with and without disintegrant. (A) Profile for clopidogrel; (B) profile for pravastatin. 


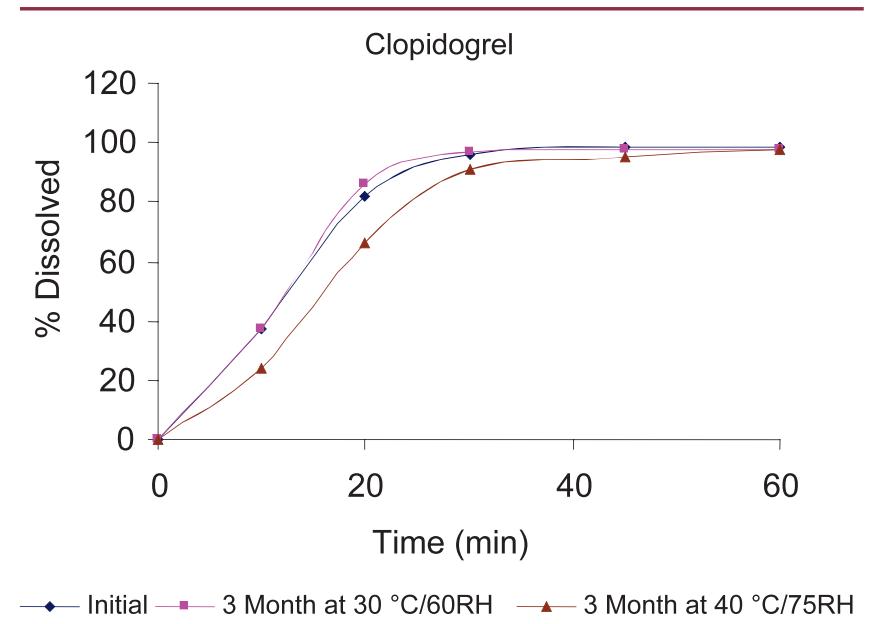

(A)

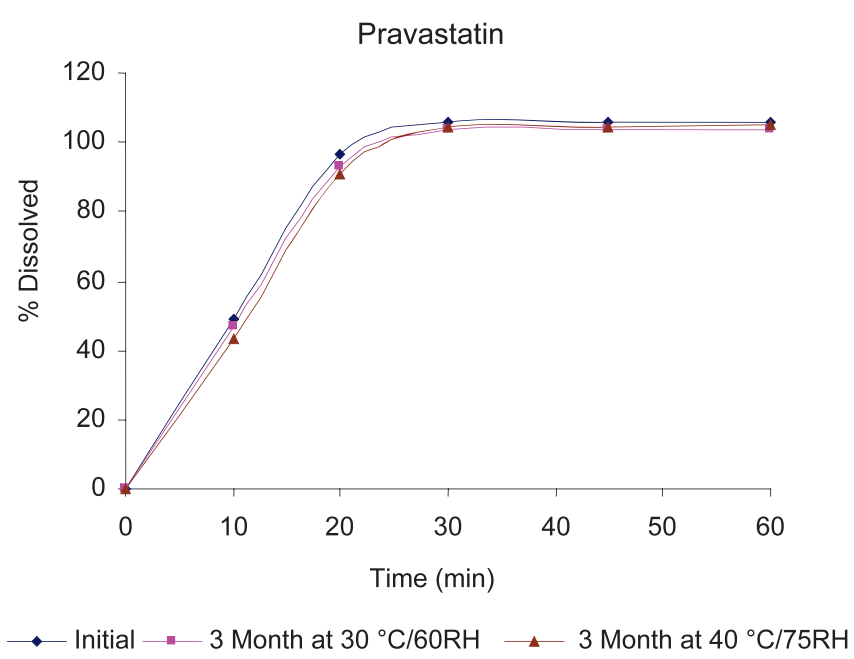

(B)

Figure 5. Dissolution profiles of initial and 3-month stability samples of clopidogrel-pravastatin combination tablets $(75 / 80 \mathrm{mg})$. (A) Profile for clopidogrel; (B) profile for pravastatin.

\section{Discriminatory Power of the Dissolution Method}

Several tablet batches manufactured under different conditions were tested to evaluate the discriminating ability of the dissolution method. The effects of tablet hardness and granulation process (fluid bed vs. roller compaction) are shown in Figure 3 (mean, $n=3$ ). As expected, the dissolution of both drugs was slightly faster for tablets with lesser hardness. Tablets granulated using roller compaction showed slower dissolution for both drugs. At the 30-min time point, the dissolution of clopidogrel and pravastatin from roller-compaction granulated tablets was $8 \%$ and $4 \%$ lower than that from fluid-bed granulated tablets, respectively. The dissolution method was also able to distinguish tablets with and without disintegrant, as shown in Figure 4 (mean, $n=6$ ).

Tablet samples stored under accelerated stability conditions were also used to evaluate the discriminatory ability of the method. As shown in Figure 5, clopidogrel dissolution at the 30-min time point for a three-month stability sample stored at $40{ }^{\circ} \mathrm{C}$ and $75 \%$ relative humidity $(75 \mathrm{RH})$ was $10 \%$ lower than that for a three-month $30^{\circ} \mathrm{C} / 60 \mathrm{RH}$ sample. The reason for the slowdown was not investigated. No further investigation was conducted. The dissolution profiles of pravastatin showed little difference between the three-month samples under each accelerated condition. Therefore, this dissolution method was capable of distinguishing changes in composition or manufacturing process as well as on stability.

The dissolution profile data were also compared mathematically using the similarity fit factor $f_{2}$, which is calculated as follows:

$$
f_{2}=50 \cdot \log \left\{\left[1+\frac{1}{n} \sum_{t=1}^{n} w_{t}\left(R_{t}-T_{t}\right)^{2}\right]^{-0.5} \times 100\right\}
$$

where $R_{t}$ and $T_{t}$ are the average values of the two data sets at time point $t$ and $n$ is the total number of time points used for calculation. The concept of the $f_{2}$ approach was described by Moore and Flanner (16). Two profiles are considered dissimilar when $f_{2}$ is less than 50 . The similarity increases as the $f_{2}$ value increases above 50 and approaches 100 . It is recommended that only one point past the plateau of the profiles be used to calculate this factor. Table 3 lists the $f_{2}$ values used for comparison of the dissolution profiles $(n=6)$ for each of the process parameters evaluated. These results confirm that the dissolution test procedure has discriminating power for the granulating process, the presence or absence of disintegrant, and the storage at accelerated stability

Table 3. Similarity Factors $\left(f_{2}\right)$ for Dissolution Profiles of Tablets with Different Parameters

\begin{tabular}{|c|c|c|c|c|c|}
\hline \multirow[b]{2}{*}{$\begin{array}{l}\text { Process } \\
\text { parameter }\end{array}$} & \multicolumn{5}{|c|}{$f_{2}$ value } \\
\hline & $\begin{array}{l}\text { Granulation by fluid bed } \\
\text { vs. roller compaction } \\
\text { (hardness of } 19 \mathrm{SCU} \text { ) }\end{array}$ & $\begin{array}{l}\text { Tablets with hardness of } \\
19 \text { SCU vs. } 27 \text { SCU (fluid } \\
\text { bed granulation) }\end{array}$ & $\begin{array}{l}\text { Tablets with hardness of } \\
19 \text { SCU vs. } 31 \text { SCU (roller } \\
\text { compaction granulation) }\end{array}$ & $\begin{array}{l}\text { Tablets containing } \\
\text { disintegrant vs. no } \\
\text { disintegrant }\end{array}$ & $\begin{array}{c}\text { Tablets stored for } 3 \\
\text { months at } 30^{\circ} \mathrm{C} / 60 \% \mathrm{RH} \\
\text { vs. } 40^{\circ} \mathrm{C} / 75 \% \mathrm{RH}\end{array}$ \\
\hline Clopidogrel & 44 & 60 & 72 & 36 & 42 \\
\hline Pravastatin & 43 & 80 & 56 & 35 & 79 \\
\hline
\end{tabular}

SCU $=$ Strong-Cobb units 


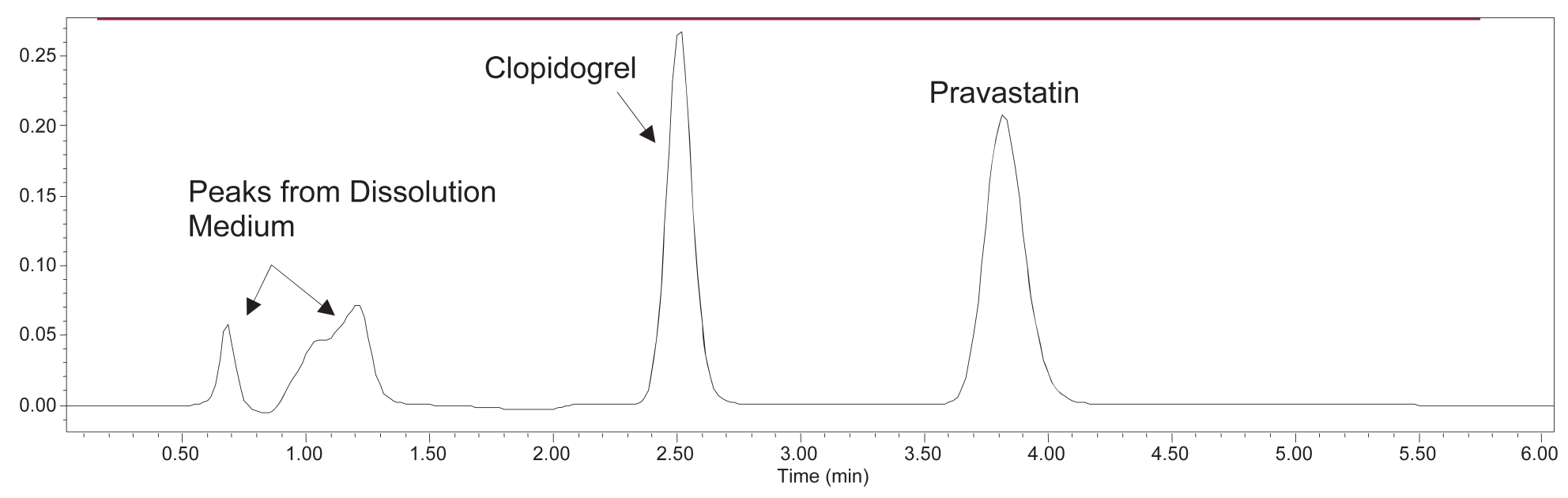

Figure 6. Chromatogram of clopidogrel and pravastatin in the dissolution medium.

conditions for clopidogrel. For pravastatin, the dissolution test is discriminative for the granulation process and the presence or absence of disintegrant. Even though $f_{2}$ values were greater than 50 for differences in hardness, the relatively low $f_{2}$ indicates at least some discrimination for this parameter.

\section{Optimization of Chromatographic Conditions for Sample Analysis}

Since direct UV spectrophotometric analysis of the dissolution samples afforded no specificity for clopidogrel or pravastatin, an HPLC method was developed to provide adequate specificity and a reasonably short run time and to eliminate surfactant interference. To accommodate the wide difference in polarity and $\mathrm{pH}$-dependent stability of the two drugs, many column types and different mobile phases were investigated to achieve the desired specificity and short run time. The optimized chromatographic separation is shown in Figure 6. These parameters provided satisfactory separation of clopidogrel and pravastatin from each other and from the tablet excipients and medium additives. Several peaks attributed to CTAB impurities eluted before the clopidogrel peak but did not interfere with the determination of either drug.

It must be noted that chromatographic conditions had to be modified continuously during the course of altering the dissolution medium in method development because the presence of SDS and Triton in the dissolution samples affected the selectivity and peak shape.

\section{Method Validation}

The HPLC method for analyzing the dissolution samples was validated according to current ICH and FDA guidelines. The validation included specificity, linearity, accuracy, precision, and robustness studies. The validation figures of merit are summarized in Table 4. The results of the validation show that the method is acceptable for use in a regulated laboratory.
Table 4. Validation Results

LINEAR REGRESSION

\begin{tabular}{lcc}
\hline & $\begin{array}{c}\text { Range (\% of nominal assay } \\
\text { concentration) }\end{array}$ & $\begin{array}{c}\text { Correlation } \\
\text { Coefficient }\end{array}$ \\
\hline Clopidogrel & $10-200$ & 0.9999 \\
Pravastatin & $6.25-125$ & 0.9999
\end{tabular}

ACCURACY

\begin{tabular}{lccc}
\hline & \multicolumn{4}{c}{ \% of nominal assay concentration } \\
\cline { 2 - 4 } Clopidogrel & $\mathbf{8 0}$ & $\mathbf{1 0 0}$ & $\mathbf{1 2 0}$ \\
\hline Average (3) & 99.8 & 100.7 & 100.5 \\
\%RSD (3) & 0.51 & 0.70 & 0.85 \\
\hline & \multicolumn{2}{c}{$\%$ of nominal assay concentration } \\
\cline { 2 - 4 } Pravastatin & $\mathbf{7 5}$ & $\mathbf{1 0 0}$ & $\mathbf{1 2 5}$ \\
\hline Average (3) & 100.4 & 101.1 & 101.0 \\
\%RSD (3) & 0.70 & 0.35 & 0.25
\end{tabular}

FILTER EVALUATION

\begin{tabular}{|c|c|c|}
\hline & \multicolumn{2}{|c|}{$\begin{array}{l}\text { Response of filtered versus unfiltered } \\
\text { standard solution }\end{array}$} \\
\hline & $0.8-\mu \mathrm{m}$ Millex AA Filter & $0.45-\mu \mathrm{m}$ PVDF Filter \\
\hline Clopidogrel & $100.1 \%$ & $100.0 \%$ \\
\hline Pravastatin & $99.9 \%$ & $100.5 \%$ \\
\hline \multicolumn{3}{|c|}{ SOLUTION STABILITY STUDY } \\
\hline & \multicolumn{2}{|c|}{$\begin{array}{l}\text { Assay of stored solution versus a freshly } \\
\text { prepared standard solution }\end{array}$} \\
\hline & after 2 days & after 12 days \\
\hline Clopidogrel & $100.6 \%$ & $99.6 \%$ \\
\hline Pravastatin & $100.4 \%$ & $98.1 \%$ \\
\hline
\end{tabular}




\section{CONCLUSIONS}

A single dissolution method that overcame competing stability and solubility challenges was developed for a clopidogrel-pravastatin combination tablet. A screening study was conducted to optimize the dissolution parameters. The optimal dissolution conditions, including a medium at $\mathrm{pH} 5.5$ with a cationic surfactant (CTAB), can accommodate the testing of two drugs in a single run and increase laboratory efficiency. A robust and discriminating HPLC method for analyzing clopidogrel and pravastatin simultaneously in dissolution samples was developed and was successfully validated.

\section{ACKNOWLEDGMENTS}

The authors gratefully acknowledge the valuable insights provided by Pankaj Shah, Joan Ruan, Steven Klohr, and John Grosso of the Bristol Myers Squibb Co. in their review of this article.

\section{REFERENCES}

1. Chapman, M. J. From pathophysiology to targeted therapy for atherothrombosis: a role for the combination of statin and aspirin in secondary prevention. Pharmacol. Therapeut. 2007, 113 (1), 184-196.

2. Hennekens, C. H.; Sacks, F. M.; Tonkin, A.; Jukema, J.W.; Byington, R. P.; Pitt, B.; Berry, D. A.; Berry, S. M.; Ford, N. F.; Walker, A. J.; Natarajan, K.; Sheng-Lin, C.; Fiedorek, F.T.; Belder, R. Additive benefits of pravastatin and aspirin to decrease risks of cardiovascular disease. Arch. Intern. Med. 2004, 164 (1), 40-44.

3. Wenaweser, P.;Windecker, S.; Billinger, M. Effect of atorvastatin and pravastatin on platelet inhibition by aspirin and clopidogrel treatment in patients with coronary stent thrombosis. Am. J. Cardiol. 2007, 99 (3), 353-356.

4. Harker, L. A.; Boissel, J-P.; Pilgrim, A. J.; Gent M. Comparative Safety and Tolerability of Clopidogrel and Aspirin: Results from CAPRIE. Drug Safety 1999, 21 (4), 325-335.

5. CAPRIE Steering Committee. A randomised, blinded, trial of clopidogrel versus aspirin in patients at risk of ischaemic events (CAPRIE). Lancet 1996, 348 (9038), 1329-1339.
6. Jacobson, A. K. Platelet ADP receptor antagonists: ticlopidine and clopidogrel. Best Pract. Res. Clin. Haematol. 2004, 17 (1), 55-64.

7. Hirano, T.; Komuro F.; Furukawa, S.; Nagano, S.; Takahashi T. Effect of pravastatin sodium, a new inhibitor of 3-hydroxy-3-methylglutaryl coenzyme A reductase, on very-low-density lipoprotein composition and kinetics in hyperlipidemia associated with experimental nephrosis. Metab. 1990, 39 (6),605-609.

8. Singh, S. S.; Sharma, K.; Barot, D.; Mohan, P. R.; Lohray, V.B. Estimation of carboxylic acid metabolite of clopidogrel in Wistar rat plasma by HPLC and its application to a pharmacokinetic study.J. Chromatogr. $B$ 2005, 821 (2), 173-180.

9. Ksycinska, H.; Rudzki, P.; Bukowska-Kiliszek, M. Determination of clopidogrel metabolite (SR26334) in human plasma by LC-MS.J. Pharm. Biomed. Anal. 2006, $41(2), 533-539$.

10. Gomez, Y.; Adams, E.; Hoogmartens, J. Analysis of purity in 19 drug product tablets containing clopidogrel: 18 copies versus the original brand. J. Pharm. Biomed. Anal. 2004, 34 (2), 341-348.

11. Mitakos, A.; Panderi, I. A validated LC method for the determination of clopidogrel in pharmaceutical preparations. J. Pharm. Biomed. Anal. 2002, 28 (3-4), 431-438.

12. Otter, K.; Mignat, C. Determination of pravastatin in human plasma by high-performance liquid chromatography with ultraviolet detection. J. Chromatogr. B 1998, 708 (1), 235-241.

13. Kocijan, A.; Grahek, R.; Bastarda, A.; Kralj, L. Fast analysis of pravastatin in production media. J. Chromatogr. $B$ 2005, 822 (1-2), 311-315.

14. Reagents, Indicators, and Solutions. In United States Pharmacopeia and National Formulary USP 29-NF 24; The United States Pharmacopeial Convention, Inc.: Rockville, MD, 2006.

15. <711> Dissolution. In United States Pharmacopeia and National Formulary USP 29-NF 24, The United States Pharmacopeial Convention, Inc.: Rockville, MD, 2006; p 2673.

16. Moore, J.; Flanner, H. Mathematical comparison of curves with an emphasis on in vitro dissolution profiles. Pharm. Technol. 1996, 20 (6), 64-74. 